\title{
Generalized Alcuin's Sequence
}

\author{
Daniel Panario, Murat Sahin and Qiang Wang * \\ School of Mathematics and Statistics \\ Carleton University \\ Ottawa, K1S 5B6, Canada \\ \{daniel, msahin, wang\}@math. carleton.ca
}

Submitted: Oct 12, 2012; Accepted: Dec 18, 2012; Published: Dec 31, 2012

Mathematics Subject Classifications: 11B50, 11P81, 05A15

\begin{abstract}
We introduce a new family of sequences $\left\{t_{k}(n)\right\}_{n=-\infty}^{\infty}$ for given positive integer $k \geqslant 3$. We call these new sequences as generalized Alcuin's sequences because we get Alcuin's sequence which has several interesting properties when $k=3$. Also, $\left\{t_{k}(n)\right\}_{n=0}^{\infty}$ counts the number of partitions of $n-k$ with parts being $k$, $(k-1), 2(k-1), 3(k-1), \ldots,(k-1)(k-1)$. We find an explicit linear recurrence equation and the generating function for $\left\{t_{k}(n)\right\}_{n=-\infty}^{\infty}$. For the special case $k=4$ and $k=5$, we get a simpler formula for $\left\{t_{k}(n)\right\}_{n=-\infty}^{\infty}$ and investigate the period of $\left\{t_{k}(n)\right\}_{n=-\infty}^{\infty}$ modulo a fixed integer. Also, we get a formula for $p_{5}(n)$ which is the number of partitions of $n$ into exactly 5 parts.
\end{abstract}

Keywords: Alcuin's sequence, integer partition.

\section{Introduction}

Alcuin of York (c. 740-804) lived over four hundred years before Fibonacci. Like Fibonacci, Alcuin has a sequence of integers named after him. Alcuin's sequence $\left\{t_{3}(n)\right\}_{n=0}^{\infty}$ has several interesting properties and it can be defined as the number of incongruent integer triangles of perimeter $n$. It is also related to the solutions to the flask-sharing problem (see pages 150 to 165 of [4]) and to $p_{3}(n)$, the number of partitions of $n$ into exactly 3 parts. If $n$ is even then $t_{3}(n)=p_{3}\left(\frac{n}{2}\right)$, else $t_{3}(n)=t_{3}(n+3)$; see [3]. So we can write,

$$
t_{3}(n)= \begin{cases}p_{3}\left(\frac{n}{2}\right), & \text { if } n \equiv 0(\bmod 2), \\ p_{3}\left(\frac{n+3}{2}\right), & \text { if } n \equiv 1(\bmod 2) .\end{cases}
$$

${ }^{*}$ The authors were supported by NSERC of Canada. 
We now introduce a new sequence $\left\{t_{k}(n)\right\}_{n=0}^{\infty}$ for given positive integer $k \geqslant 3$ that is a generalization of the Alcuin's sequence:

$$
t_{k}(n)=p_{k}\left(\frac{n+a_{i}}{k-1}\right), \quad \text { if } n \equiv i(\bmod k-1)
$$

where if $i=0$ then $a_{i}=0$ else $a_{i}=k(k-1-i)$ and $p_{k}(x)$ is the number of partitions of $x$ into exactly $k$ parts. We call $\left\{t_{k}(n)\right\}_{n=0}^{\infty}$ a generalized Alcuin's sequence. Later on, we extend this definition so that $t_{k}(n)$ ranges from $n=-\infty$ to $\infty$.

In Section 2, we find an explicit linear recurrence equation and the generating function for $\left\{t_{k}(n)\right\}_{n=-\infty}^{\infty}$. From the generating function, we can see that $t_{k}(n)$ counts the number of partitions of $n-k$ with parts being $k,(k-1), 2(k-1), \ldots,(k-1)(k-1)$. In Sections 3 and 4, we obtain simpler formulae for $\left\{t_{k}(n)\right\}_{n=-\infty}^{\infty}$ with $k=4$ and $k=5$, respectively, and using these formulae we study the period of $\left\{t_{k}(n)\right\}_{n=-\infty}^{\infty}$ modulo a fixed integer for the special cases $k=4$ and $k=5$. For $k=3$ the Alcuin sequence modulo $m$ has least period $12 m$ [3]. Motivated by this result we study the least period of generalized Alcuin's sequences for bigger $k$. For any integer $m \geqslant 2$, the sequence $\left\{t_{4}(n)(\bmod m)\right\}_{n=-\infty}^{\infty}$ is periodic with the least period $36 \mathrm{~m}$. Similarly, for $k=5$, the least period is either $240 \mathrm{~m}$ for odd $m$, or $480 m$ for even $m$. As a by-product, we also obtain a formula for $p_{5}(n)$, the number of partitions of $n$ into exactly 5 parts. Conclusions and further work are given in Section 5.

\section{Generalization of Alcuin's Sequence}

Definition 1 (Generalized Alcuin's Sequence). Let us define the sequence $\left\{t_{k}(n)\right\}_{n=0}^{\infty}$

$$
t_{k}(n)=p_{k}\left(\frac{n+a_{i}}{k-1}\right), \quad \text { if } n \equiv i(\bmod k-1)
$$

where if $i=0$ then $a_{i}=0$ else $a_{i}=k(k-1-i)$ and $p_{k}(x)$ is the number of partitions of $x$ into exactly $k$ parts.

As we will see later, $t_{k}(n)$ is the number of partitions of $n-k$ such that the parts are $k,(k-1), 2(k-1), 3(k-1), \ldots,(k-1)(k-1)$.

If we take $k=3$ in Definition 1 , we get Alcuin's sequence :

$$
t_{3}(n)= \begin{cases}p_{3}\left(\frac{n}{2}\right), & \text { if } n \equiv 0(\bmod 2), \\ p_{3}\left(\frac{n+3}{2}\right), & \text { if } n \equiv 1(\bmod 2) .\end{cases}
$$

Obviously, we can get

$$
t_{k}(n(k-1))=p_{k}(n)
$$


and, for $1 \leqslant i \leqslant k-2$,

$$
\begin{aligned}
t_{k}(n(k-1)+i) & =p_{k}\left(\frac{n(k-1)+i+a_{i}}{k-1}\right)=p_{k}\left(\frac{n k-n+i+k^{2}-k-k i}{k-1}\right) \\
& =p_{k}\left(\frac{(n+k-i)(k-1)}{k-1}\right)=p_{k}(n+k-i) .
\end{aligned}
$$

We use these facts in the following.

Theorem 2. The generating function of the sequence $\left\{t_{k}(n)\right\}_{n=0}^{\infty}$ is

$$
F_{k}(x)=\frac{x^{k}}{\left(1-x^{k}\right) \prod_{i=1}^{k-1}\left(1-x^{i(k-1)}\right)} .
$$

Proof. The generating function for $\left\{t_{k}(n)\right\}_{n=0}^{\infty}$ is

$$
F_{k}(x)=\sum_{n=0}^{\infty} t_{k}(n) x^{n} .
$$

Then we can write, using Equations (1) and (2),

$$
\begin{aligned}
F_{k}(x) & =\sum_{n=0}^{\infty} \sum_{i=0}^{k-2} t_{k}(n(k-1)+i) x^{n(k-1)+i} \\
& =\sum_{n=0}^{\infty}\left(p_{k}(n) x^{n(k-1)}+\sum_{i=1}^{k-2} p_{k}(n+k-i) x^{n(k-1)+i}\right) .
\end{aligned}
$$

Formulas for the generating functions of the sequences $\left\{p_{k}(n)\right\}$ are well known (see [2], for example):

$$
G_{k}(x)=\sum_{n=0}^{\infty} p_{k}(n) x^{n}=\frac{x^{k}}{\prod_{i=1}^{k}\left(1-x^{i}\right)} .
$$

Then, for $0 \leqslant j \leqslant k-1$, we derive

$$
G_{k}^{j}(x)=\sum_{n=0}^{\infty} p_{k}(n+j) x^{n}=\frac{x^{k-j}}{\prod_{i=1}^{k}\left(1-x^{i}\right)} .
$$

Substituting in Equation (3), we get

$$
\begin{aligned}
F_{k}(x) & =G_{k}^{0}\left(x^{k-1}\right)+\sum_{i=1}^{k-2} x^{i} G_{k}^{k-i}\left(x^{k-1}\right) \\
& =\frac{x^{k}\left(1+x^{k}+x^{2 k}+\cdots+x^{(k-2) k}\right)}{\prod_{i=1}^{k}\left(1-x^{i(k-1)}\right)} .
\end{aligned}
$$

The proof is complete using the fact that

$$
1-x^{k(k-1)}=\left(1-x^{k}\right)\left(1+x^{k}+x^{2 k}+\cdots+x^{(k-2) k}\right) .
$$


It is now clear that $t_{k}(n)$ counts the number of partitions of $n-k$ into parts $k,(k-1)$, $2(k-1), 3(k-1), \ldots,(k-1)(k-1)$.

Let $p(x)=\left(1-x^{k}\right) \prod_{i=1}^{k-1}\left(1-x^{i(k-1)}\right)$ and $c_{i}$ be the coefficient of the term $x^{i}$ in the expansion of $p(x)$. Let $\ell$ be the degree of $p(x)$; then $\ell=\frac{k\left(k^{2}-2 k+3\right)}{2}$.

Theorem 3. The sequence $\left\{t_{k}(n)\right\}_{n=0}^{\infty}$ is determined by the linear recurrence relation of order $\ell$

$$
t_{k}(n)=-\sum_{i=1}^{\ell} c_{i} t_{k}(n-i),
$$

for $n \geqslant \ell$, where $c_{i}$ 's are the coefficients of $p(x)$ defined above, and the initial values of $t_{k}(n)$ for $0 \leqslant n \leqslant \ell-1$ are obtained from Definition 1.

Proof. By the generating function of the sequence $\left\{t_{k}(n)\right\}_{n=0}^{\infty}$, we have

$$
\begin{aligned}
x^{k} & =p(x) \sum_{n=0}^{\infty} t_{k}(n) x^{n} \\
& =\sum_{n=0}^{\infty} t_{k}(n)\left(x^{n}+c_{1} x^{n+1}+c_{2} x^{n+2}+\cdots+c_{\ell} x^{n+\ell}\right) .
\end{aligned}
$$

The linear recurrence relation of order $\ell$ can be read off by equating coefficients of $x^{n}$ for $n \geqslant \ell$.

Let us use the same recurrence relation in Theorem 3 to define $t_{k}(n)$ for $n<0$, so this extends the ranges from $n=-\infty$ to $\infty$.

Corollary 4. If we take $k=3$ in the sequence $\left\{t_{k}(n)\right\}_{n=-\infty}^{\infty}$ we get as special case the Alcuin's sequence in [3]. Also, substituting $k=3$ in Theorem 2 and 3, we get Theorem 2 and Theorem 3 in [3], respectively.

\section{The case $k=4$}

Let us take $k=4$ in the generalized Alcuin's sequences for $\left\{t_{k}(n)\right\}_{n=0}^{\infty}$ given in Definition 1 . We obtain the sequence $\left\{t_{4}(n)\right\}_{n=0}^{\infty}$

$$
t_{4}(n)= \begin{cases}p_{4}\left(\frac{n}{3}\right), & \text { if } n \equiv 0(\bmod 3) \\ p_{4}\left(\frac{n+8}{3}\right), & \text { if } n \equiv 1(\bmod 3) \\ p_{4}\left(\frac{n+4}{3}\right), & \text { if } n \equiv 2(\bmod 3) .\end{cases}
$$

If we substitute $k=4$ in Theorem 2 and Theorem 3, we get the generating function

$$
F_{4}(x)=\frac{x^{4}}{\left(1-x^{4}\right)\left(1-x^{3}\right)\left(1-x^{6}\right)\left(1-x^{9}\right)}
$$


and the linear recurrence relation of order 22

$$
\begin{aligned}
t_{4}(n)= & t_{4}(n-3)+t_{4}(n-4)+t_{4}(n-6)-t_{4}(n-7)-t_{4}(n-10)-t_{4}(n-12)(5) \\
& -t_{4}(n-15)+t_{4}(n-16)+t_{4}(n-18)+t_{4}(n-19)-t_{4}(n-22)
\end{aligned}
$$

for $n \geqslant 22$, together with the initial values $0,0,0,0,1,0,0,1,1,0,2,1,1,3,2,1,5,3,2,6,5,3$ of $t_{4}(n)$ for $0 \leqslant n \leqslant 21$.

Example 5. From the generating function $F_{4}(x), t_{4}(n)$ is the number of partitions of $n-4$ with parts $3,4,6$ and 9 . For example, $t_{4}(16)=5$ and 12 can be partitioned in five distinct ways:

$$
3+3+3+3, \quad 4+4+4, \quad 3+3+6, \quad 6+6 \quad \text { and } 3+9 .
$$

Theorem 6. Let $\|x\|$ be the nearest integer to $x$. Then for all $n \geqslant 0$,

$$
p_{4}(n)= \begin{cases}\left\|\frac{(n+1)^{3}}{144}-\frac{(n+1)}{48}\right\|, & \text { if } n \text { is even, } \\ \left\|\frac{(n+1)^{3}}{144}-\frac{(n+1)}{12}\right\|, & \text { if } n \text { is odd. }\end{cases}
$$

Proof. The generating function of the numbers $p_{4}(n)$ is

$$
G_{4}(x)=\frac{x^{4}}{(1-x)\left(1-x^{2}\right)\left(1-x^{3}\right)\left(1-x^{4}\right)} .
$$

Now, by partial fractions we obtain

$$
\begin{aligned}
G_{4}(x)= & -\frac{13}{288(x-1)^{2}}+\frac{1}{24(x-1)^{3}}+\frac{1}{32(x+1)^{2}}+\frac{1}{24(x-1)^{4}} \\
& +\frac{1}{8\left(x^{2}+1\right)}-\frac{1}{9\left(x^{2}+x+1\right)} .
\end{aligned}
$$

Using the general binomial theorem (see [2], for example), the first four terms of $G_{4}(x)$ can be written as

$$
-\frac{13}{288} \sum_{n=0}^{\infty}(n+1) x^{n}-\frac{1}{24} \sum_{n=0}^{\infty}\left(\begin{array}{c}
n+2 \\
n
\end{array}\right) x^{n}+\frac{1}{32} \sum_{n=0}^{\infty}(n+1)(-1)^{n} x^{n}+\frac{1}{24} \sum_{n=0}^{\infty}\left(\begin{array}{c}
n+3 \\
n
\end{array}\right) x^{n} .
$$

Thus the first four terms give the following coefficient of $x^{n}$ :

$$
\frac{2 n^{3}+6 n^{2}-9 n+9 n(-1)^{n}+9(-1)^{n}-13}{288} .
$$


The last two terms in Equation (6) can be handled by Maple using Taylor expansions and their contribution to the coefficient of $x^{n}$ is $c / 72$, where $c$ is given in the following table.

\begin{tabular}{c|cccccccccccc}
$n \bmod 12$ & 0 & 1 & 2 & 3 & 4 & 5 & 6 & 7 & 8 & 9 & 10 & 11 \\
\hline$c$ & 1 & 8 & -9 & -8 & 17 & 0 & -17 & 8 & 9 & -8 & -1 & 0
\end{tabular}

Combining all the contributions, we obtain the formula $p_{4}(n)=\frac{n^{3}}{144}+\frac{n^{2}}{48}-\frac{c-1}{72}$, for $n$ even, and $p_{4}(n)=\frac{n^{3}}{144}+\frac{n^{2}}{48}-\frac{n}{16}+\frac{2 c-11}{144}$, for $n$ odd. This can be represented as in the statement of the theorem.

Remark 7. A search in internet [5] gives the previous formula for $p_{4}(n)$, but we could not find any proof of it. For the sake of completeness we give such a proof in the previous theorem using generating functions.

Plugging the formula for $p_{4}(n)$ into the definition of $t_{4}(n)$ we obtain the following corollary.

Corollary 8. Let $C=1 / 3888$ and $\|x\|$ be the nearest integer to $x$. Then for all $n \geqslant 0$,

$$
t_{4}(n)= \begin{cases}\left\|C\left(n^{3}+9 n^{2}-54\right)\right\|, & \text { if } n \equiv 0(\bmod 6), \\ \left\|C\left(n^{3}+33 n^{2}+255 n+143\right)\right\|, & \text { if } n \equiv 1(\bmod 6), \\ \left\|C\left(n^{3}+21 n^{2}+120 n+154\right)\right\|, & \text { if } n \equiv 2(\bmod 6), \\ \left\|C\left(n^{3}+9 n^{2}-81 n-297\right)\right\|, & \text { if } n \equiv 3(\bmod 6), \\ \left\|C\left(n^{3}+33 n^{2}+336 n+1034\right)\right\|, & \text { if } n \equiv 4(\bmod 6), \\ \left\|C\left(n^{3}+21 n^{2}+39 n-413\right)\right\|, & \text { if } n \equiv 5(\bmod 6) .\end{cases}
$$

Let us use the same recurrence relation in (5) to define $\left\{t_{4}(n)\right\}_{n=-\infty}^{\infty}$ for $n<0$. Then we get

$$
\begin{aligned}
t_{4}(n)= & t_{4}(n+3)+t_{4}(n+4)+t_{4}(n+6)-t_{4}(n+7)-t_{4}(n+10)-t_{4}(n+12)(7) \\
& -t_{4}(n+15)+t_{4}(n+16)+t_{4}(n+18)+t_{4}(n+19)-t_{4}(n+22)
\end{aligned}
$$

together with the initial values of $t_{4}(n)$ for $0 \leqslant n \leqslant 21$. Recall these initial values are $0,0,0,0,1,0,0,1,1,0,2,1,1,3,2,1,5,3,2,6,5,3$. From this equation we see that recurrence relation (5) also holds for any integer $n$.

By using Equation (7) we can construct the following table of values of $t_{4}(n)$ when $-22 \leqslant n \leqslant 8$ :

\begin{tabular}{c|ccccccccccccccccc}
$n$ & -22 & -21 & -20 & -19 & -18 & -17 & -16 & -15 & $\ldots$ & 1 & 2 & 3 & 4 & 5 & 6 & 7 & 8 \\
\hline$t_{4}(n)$ & -1 & -1 & 0 & 0 & -1 & 0 & 0 & 0 & $\ldots$ & 0 & 0 & 0 & 1 & 0 & 0 & 1 & 1
\end{tabular}

Obviously, the sequence is palindromic from the table if we ignore signs; we can easily see that

$$
t_{4}(-n)=-t_{4}(n-14)
$$

Note that $\ell-2 k=22-8=14$ for the sequence $\left\{t_{4}(n)\right\}_{n=-\infty}^{\infty}$, where $k=4$ and $\ell=22$. 
Theorem 9. For any integer $m \geqslant 2$, the sequence $\left\{t_{4}(n)(\bmod m)\right\}_{n=-\infty}^{\infty}$ is periodic with least period $36 \mathrm{~m}$.

Proof. Let $L$ be the least period of $\left\{t_{4}(n)(\bmod m)\right\}_{n=-\infty}^{\infty}$. First we prove that $36 m$ is a period of the sequence and thus $L$ divides $36 m$. That is, for each $m \geqslant 2$,

$$
t_{4}(n+36 m) \equiv t_{4}(n)(\bmod m) \quad \text { for any integer } n .
$$

In order to show this, we break it into 5 cases.

(1) $n \geqslant 0:$ For $n \equiv 0(\bmod 6)$, and by Corollary 8 ,

$$
\begin{aligned}
t_{4}(n+36 m) & =\left\|\frac{n^{3}}{3888}+\frac{n^{2}}{432}-\frac{1}{72}\right\|+\frac{n^{2}}{36} m+\frac{n}{6} m+n m^{2}+12 m^{3}+3 m^{2} \\
& \equiv t_{4}(n)(\bmod m) .
\end{aligned}
$$

In a similar way one shows that, Equation (9) is also satisfied for $n \equiv 1,2,3,4,5$ $(\bmod 6)$.

(2) $-14<n<0$ : Equation (9) is also satisfied for $n=-1,-2,-3, \ldots,-13$ by using Corollary 8 and Equation (8).

(3) $-36 m<n \leqslant-14:$ For $n \equiv 0(\bmod 6)$, and using again Corollary 8 and Equation (8),

$$
\begin{aligned}
t_{4}(n+36 m) & =-\left\|-\frac{n^{3}}{3888}-\frac{n^{2}}{432}+\frac{1}{72}\right\|+\frac{n^{2}}{36} m+\frac{n}{6} m+n m^{2}+12 m^{3}+3 m^{2} \\
& \equiv-t_{4}(-n-14)(\bmod m) \\
& \equiv t_{4}(n)(\bmod m) .
\end{aligned}
$$

Again, in a similar way, Equation $(9)$ is also satisfied for $n \equiv 1,2,3,4,5(\bmod 6)$.

(4) $-36 m-14<n \leqslant-36 m$ : Since $n=-36 m-a$ for $0 \leqslant a \leqslant 13$, we have

$$
t_{4}(n+36 m)=t_{4}(-36 m-a+36 m)=t_{4}(-a) \equiv 0(\bmod m)
$$

and

$$
\begin{aligned}
t_{4}(n) & =t_{4}(-36 m-a)=t_{4}(36 m+a-14) \quad(\text { by Equation }(8)) \\
& \equiv 0(\bmod m),
\end{aligned}
$$

so that $t_{4}(n+36 m) \equiv t_{4}(n)(\bmod m)$ for $-36 m-14<n \leqslant-36 m$. 
(5) $n \leqslant-36 m-14:$ For $n \equiv 0(\bmod 6)$, and using Corollary 8 and Equation $(8)$,

$$
\begin{aligned}
t_{4}(n+36 m) & =-t_{4}(-n-36 m-14) \\
& =-\left\|-\frac{n^{3}}{3888}-\frac{n^{2}}{432}+\frac{1}{72}\right\|+\frac{n^{2}}{36} m+\frac{n}{6} m+n m^{2}+12 m^{3}+3 m^{2} \\
& \equiv-t_{4}(-n-14)(\bmod m) \\
& \equiv t_{4}(n)(\bmod m) .
\end{aligned}
$$

As before, Equation (9) is also satisfied for $n \equiv 1,2,3,4,5(\bmod 6)$.

Hence, Equation (9) is satisfied for any integer $n$. Therefore, $36 \mathrm{~m}$ is a period of the sequence and $L$ divides $36 \mathrm{~m}$.

Secondly, we prove $L=36 \mathrm{~m}$. This can be verified by a computer program for small $m \leqslant 10$. Thus, here we prove $L=36 m$ for only $m>10$. Let $\lambda=L, L-1$ or $L-2$ such that $\lambda \equiv 0(\bmod 3)$. First of all, we assume that $\lambda$ is even. Since $t_{4}(-2)=t_{4}(-1)=$ $t_{4}(0)=t_{4}(1)=t_{4}(2)=t_{4}(3)=0$, we have

$$
t_{4}(\lambda) \equiv t_{4}(\lambda+1) \equiv t_{4}(\lambda+2) \equiv t_{4}(\lambda+3) \equiv 0(\bmod m) .
$$

Hence, $m \mid M$ where $M=\left[t_{4}(\lambda+1)-t_{4}(\lambda+2)\right]+\left[t_{4}(\lambda)-t_{4}(\lambda+3)\right]$.

We observe that $\left\{t_{4}(n)(\bmod m)\right\}_{n=-\infty}^{\infty}$ is an aperiodic pattern for $-188 \leqslant n \leqslant 174$ with the aid of a computer program for $m>1495$. In addition to this, we can also show using a computer that $L \geqslant 362$ for $10<m \leqslant 1495$. Therefore, we have $L \geqslant 362$ which means that $\lambda \geqslant 360$.

By the definition of nearest integer function, we have

$$
\|x \mp y\|=\|x\| \mp\|y\|+\alpha, \quad \alpha=0,1 \text { or }-1
$$

and

$$
\|x\|+\|y\|<x+y+1
$$

for any $x$ and $y$. From (10), we get

$$
\begin{aligned}
t_{4}(\lambda)+t_{4}(\lambda+1) & =\left\|\frac{2 \lambda^{3}}{3888}+\frac{5 \lambda^{2}}{432}+\frac{\lambda}{12}+\frac{7}{72}\right\|+\alpha_{1}, \quad-1 \leqslant \alpha_{1} \leqslant 1 \\
& =\left\|\frac{2 \lambda^{3}}{3888}\right\|+\left\|\frac{5 \lambda^{2}}{432}\right\|+\left\|\frac{\lambda}{12}\right\|+\left\|\frac{7}{72}\right\|+\alpha_{2}, \quad-4 \leqslant \alpha_{2} \leqslant 4
\end{aligned}
$$

and

$$
\begin{aligned}
t_{4}(\lambda+2)+t_{4}(\lambda+3) & =\left\|\frac{2 \lambda^{3}}{3888}+\frac{5 \lambda^{2}}{432}+\frac{\lambda}{18}+\frac{1}{72}\right\|+\beta_{1}, \quad-1 \leqslant \beta_{1} \leqslant 1 \\
& =\left\|\frac{2 \lambda^{3}}{3888}\right\|+\left\|\frac{5 \lambda^{2}}{432}\right\|+\left\|\frac{\lambda}{18}\right\|+\left\|\frac{1}{72}\right\|+\beta_{2}, \quad-4 \leqslant \beta_{2} \leqslant 4 .
\end{aligned}
$$


Hence,

$$
\begin{aligned}
M & =\left\|\frac{\lambda}{12}\right\|-\left\|\frac{\lambda}{18}\right\|+\gamma_{1}, \quad-8 \leqslant \gamma_{1} \leqslant 8 \\
& =\left\|\frac{\lambda}{36}\right\|+\gamma_{2}, \quad-9 \leqslant \gamma_{2} \leqslant 9
\end{aligned}
$$

Since $\lambda \geqslant 360$, we have $M>1$.

Moreover, from (11), we have

$$
t_{4}(\lambda+1)-t_{4}(\lambda+2)<\frac{\lambda^{2}}{432}+\frac{\lambda}{36}-\frac{1}{72}+1
$$

and

$$
t_{4}(\lambda)-t_{4}(\lambda+3)<-\frac{\lambda^{2}}{432}+\frac{7}{72}+1,
$$

so we get $M<\frac{\lambda}{36}+\frac{6}{72}+2=\frac{\lambda+75}{36}$. This means that $m<\frac{\lambda+75}{36}$ since $m \mid M$ and $M \neq 0$, and it follows that

$$
36 m<\lambda+75 \leqslant L+75 .
$$

Because $L$ is a divisor of $36 \mathrm{~m}$, we conclude by inequality (12) that $L=36 \mathrm{~m}$ for any $m>10$. Hence, $L=36 m$ for $\lambda$ even. Similarly we can also show $L=36 m$ for $\lambda$ odd, and the proof is complete.

\section{The Case $k=5$}

Let us take $k=5$ in the generalized Alcuin's sequences for $\left\{t_{k}(n)\right\}_{n=0}^{\infty}$ given in Definition 1. We obtain the sequence $\left\{t_{5}(n)\right\}_{n=0}^{\infty}$

$$
t_{5}(n)= \begin{cases}p_{5}\left(\frac{n}{4}\right), & \text { if } n \equiv 0(\bmod 4), \\ p_{5}\left(\frac{n+15}{4}\right), & \text { if } n \equiv 1(\bmod 4), \\ p_{5}\left(\frac{n+10}{4}\right), & \text { if } n \equiv 2(\bmod 4) . \\ p_{5}\left(\frac{n+5}{4}\right), & \text { if } n \equiv 3(\bmod 4) .\end{cases}
$$

If we substitute $k=5$ in Theorem 2 and Theorem 3, we get the generating function

$$
F_{5}(x)=\frac{x^{5}}{\left(1-x^{5}\right)\left(1-x^{4}\right)\left(1-x^{8}\right)\left(1-x^{12}\right)\left(1-x^{16}\right)}
$$


and the linear recurrence relation of order 45

$$
\begin{aligned}
t_{5}(n)= & t_{5}(n-4)+t_{5}(n-5)+t_{5}(n-8)-t_{5}(n-9)-t_{5}(n-13) \\
& -2 t_{5}(n-20)+2 t_{5}(n-25)+t_{5}(n-32)+t_{5}(n-36)-t_{5}(n-37) \\
& -t_{5}(n-40)-t_{5}(n-41)+t_{5}(n-45)
\end{aligned}
$$

for $n \geqslant 45$, together with the values of $t_{5}(n)$ for $0 \leqslant n \leqslant 44$ that can be easily computed.

Theorem 10. Let $C=1 / 86400$. For all $n \geqslant 0$, we have

$$
p_{5}(n)= \begin{cases}\left\|C\left(30 n^{4}+300 n^{3}+300 n^{2}-3600 n-5224\right)\right\|, & \text { if } n \text { is even, } \\ \left\|C\left(30 n^{4}+300 n^{3}+300 n^{2}-900 n+1526\right)\right\|, & \text { if } n \text { is odd. }\end{cases}
$$

Proof. The generating function of $p_{5}(n)$ is

$$
G_{5}(x)=\frac{x^{5}}{(1-x)\left(1-x^{2}\right)\left(1-x^{3}\right)\left(1-x^{4}\right)\left(1-x^{5}\right)} .
$$

Now, by partial fractions we obtain that $G_{5}(x)$ is

$$
\begin{aligned}
& -\frac{1}{64(x+1)^{2}}-\frac{3}{128(x+1)}-\frac{5}{288(x-1)^{2}}-\frac{431}{86400(x-1)}-\frac{1}{120(x-1)^{5}} \\
& +\frac{5}{288(x-1)^{3}}-\frac{2 x+1}{27\left(x^{2}+x+1\right)}+\frac{x^{3}+2 x^{2}+3 x+4}{25\left(x^{4}+x^{3}+x^{2}+x+1\right)}+\frac{x-1}{16\left(x^{2}+1\right)} .
\end{aligned}
$$

Using the general binomial theorem, the first six terms of $G_{5}(x)$ can be written as

$$
\begin{aligned}
& -\frac{1}{64} \sum_{n=0}^{\infty}(n+1)(-1)^{n} x^{n}-\frac{3}{128} \sum_{n=0}^{\infty}(-1)^{n} x^{n}-\frac{5}{288} \sum_{n=0}^{\infty}(n+1) x^{n}-\frac{431}{86400} \sum_{n=0}^{\infty} x^{n} \\
& +\frac{1}{120} \sum_{n=0}^{\infty}\left(\begin{array}{c}
n+4 \\
n
\end{array}\right) x^{n}-\frac{5}{288} \sum_{n=0}^{\infty}\left(\begin{array}{c}
n+2 \\
n
\end{array}\right) x^{n} .
\end{aligned}
$$

Thus the first six terms give the following coefficient of $x^{n}$ :

$$
\frac{30 n^{4}+300 n^{3}+300 n^{2}-2250 n-1350 n(-1)^{n}-3375(-1)^{n}-1849}{86400} .
$$

As in the case of Theorem 6, the last three terms in Equation (15) can be handled by Maple using Taylor expansions and their contribution to the coefficient of $x^{n}$ is $c / 10800$, where $c$ follows now a pattern modulo 60 by taking the values $c=653,-157,1043,-1507$, 3203, - 307, 2003 and 1853. Combining all contributions, we get the desired result.

Plugging the formula for $p_{5}(n)$ into the definition of $t_{5}(n)$ we obtain the following corollary. 
Corollary 11. Let $C=1 / 11059200$. For all $n \geqslant 0$,

$$
t_{5}(n)= \begin{cases}\left\|C\left(15 n^{4}+600 n^{3}+2400 n^{2}-115200 n-668672\right)\right\|, & \text { if } n \equiv 0(\bmod 8), \\ \left\|C\left(15 n^{4}+1500 n^{3}+49650 n^{2}+564300 n+927703\right)\right\|, & \text { if } n \equiv 1(\bmod 8), \\ \left\|C\left(15 n^{4}+1200 n^{3}+29400 n^{2}+259200 n+897328\right)\right\|, & \text { if } n \equiv 2(\bmod 8), \\ \left\|C\left(15 n^{4}+900 n^{3}+13650 n^{2}-38700 n-1100297\right)\right\|, & \text { if } n \equiv 3(\bmod 8), \\ \left\|C\left(15 n^{4}+600 n^{3}+2400 n^{2}-28800 n+195328\right)\right\|, & \text { if } n \equiv 4(\bmod 8), \\ \left\|C\left(15 n^{4}+1500 n^{3}+49650 n^{2}+650700 n+3087703\right)\right\|, & \text { if } n \equiv 5(\bmod 8), \\ \left\|C\left(15 n^{4}+1200 n^{3}+29400 n^{2}+172800 n-830672\right)\right\|, & \text { if } n \equiv 6(\bmod 8), \\ \left\|C\left(15 n^{4}+900 n^{3}+13650 n^{2}+47700 n+195703\right)\right\|, & \text { if } n \equiv 7(\bmod 8) .\end{cases}
$$

Similar to Section 3, we use the same recurrence relation in (14) to define $\left\{t_{5}(n)\right\}_{n=-\infty}^{\infty}$ for $n<0$, obtaining

$$
t_{5}(-n)=t_{5}(n-35) .
$$

We note that $\ell-2 k=45-10=35$ for the sequence $\left\{t_{5}(n)\right\}_{n=-\infty}^{\infty}$ where $k=5$ and $\ell=45$.

Lemma 12. For any integer $n, 4 n^{3} \pm 15 n^{2}+5 n \equiv 0(\bmod 6)$ and $4 n^{3}+5 n \equiv 0(\bmod 3)$.

Proof. Since

$$
4 n^{3} \pm 15 n^{2}+5 n \equiv n^{2}-n \equiv n(n-1) \equiv 0(\bmod 2)
$$

and

$$
4 n^{3} \pm 15 n^{2}+5 n \equiv n^{3}-n \equiv n(n-1)(n-2) \equiv 0(\bmod 3)
$$

we get

$$
4 n^{3} \pm 15 n^{2}+5 n \equiv 0(\bmod 6)
$$

Similarly,

$$
4 n^{3}+5 n \equiv n^{3}-n \equiv n(n-1)(n-2) \equiv 0(\bmod 3) .
$$

Theorem 13. For any odd integer $m \geqslant 3$, the sequence $\left\{t_{5}(n)(\bmod m)\right\}_{n=-\infty}^{\infty}$ is periodic with least period $240 m$. For any even integer $m \geqslant 2$, the sequence $\left\{t_{5}(n)(\bmod m)\right\}_{n=-\infty}^{\infty}$ is periodic with least period $480 \mathrm{~m}$.

Proof. Let $L$ be the least period of $\left\{t_{5}(n)(\bmod m)\right\}_{n=-\infty}^{\infty}$. First we prove that if $m$ is odd then $240 m$ is a period of the sequence else $480 m$ is a period of the sequence so that if $m$ is odd then $L$ divides $240 m$ else $L$ divides $480 m$. That is for each $m \geqslant 2$ and any integer $n$, if $m$ is odd then

$$
t_{5}(n+240 m) \equiv t_{5}(n)(\bmod m)
$$

and else

$$
t_{5}(n+480 m) \equiv t_{5}(n)(\bmod m) \text {. }
$$

In order to prove this, as before, we break it into 5 cases. For simplicity, we only show the case $m$ is odd; the even case is analogous. 
(1) $n \geqslant 0:$ For $n \equiv 0(\bmod 8)$, using Corollary 11 and $m$ odd, we have

$$
\begin{aligned}
t_{5}( & n+240 m) \\
= & \left\|\frac{n^{4}}{737280}+\frac{n^{3}}{18432}+\frac{n^{2}}{4608}-\frac{n}{96}-\frac{653}{10800}\right\|+\frac{n^{3}}{768} m+\frac{5 n^{2}}{128} m+\frac{5 n}{48} m \\
& +\frac{15 n^{2}}{32} m^{2}+\frac{5(5 m-1)}{2} m+\frac{75 n}{8} m^{2}+75 n m^{3}+750 m^{3}+4500 m^{4} \\
= & t_{5}(n)+\frac{4 t^{3}}{6} m+\frac{15 t^{2}}{6} m+\frac{5 t}{6} m+30 t^{2} m^{2}+\frac{5(5 m-1)}{2} m+75 t m^{2} \\
& +600 t m^{3}+750 m^{3}+4500 m^{4} \quad(\text { by substituting } n=8 t) \\
\equiv & t_{5}(n)(\bmod m) \quad(\text { by Lemma } 12 \text { and } 5(5 m-1) \equiv 0(\bmod 2)) .
\end{aligned}
$$

In a similar way, we show that Equation (17) is also satisfied for $n \equiv 1,2,3,4,5,6,7$ $(\bmod 8)$.

(2) $-35<n<0$ : If $m$ is odd then Equation (17) is immediately satisfied for this range by using Corollary 11 and Equation (16).

(3) $-240 m<n \leqslant-35:$ For $n \equiv 0(\bmod 8)$, by using Corollary 11, Equation $(16)$ and $m$ is odd, we have

$$
\begin{aligned}
t_{5}( & n+240 m) \\
= & \left\|\frac{n^{4}}{737280}+\frac{n^{3}}{18432}+\frac{n^{2}}{4608}-\frac{n}{96}-\frac{653}{10800}\right\|+\frac{n^{3}}{768} m+\frac{5 n^{2}}{128} m+\frac{5 n}{48} m \\
& +\frac{15 n^{2}}{32} m^{2}+\frac{5(5 m-1)}{2} m+\frac{75 n}{8} m^{2}+75 n m^{3}+750 m^{3}+4500 m^{4} \\
= & t_{5}(-n-35)+\frac{4 t^{3}}{6} m+\frac{15 t^{2}}{6} m+\frac{5 t}{6} m+30 t^{2} m^{2}+\frac{5(5 m-1)}{2} m+75 t m^{2} \\
& +600 t m^{3}+750 m^{3}+4500 m^{4} \quad(\text { by substituting } n=8 t) \\
\equiv & t_{5}(n)(\bmod m) \quad(\text { by Lemma } 12 \text { and } 5(5 m-1) \equiv 0(\bmod 2)) .
\end{aligned}
$$

Again, Equation (17) is also satisfied for $n \equiv 1,2,3,4,5,6,7(\bmod 8)$.

(4) $-240 m-35<n \leqslant-240 m$ :

That is, $n=-240 m-a$ for $0 \leqslant a \leqslant 34$ then

$$
t_{5}(n+240 m)=t_{5}(-240 m-a+240 m)=t_{5}(-a) \equiv 0(\bmod m)
$$

and

$$
\begin{aligned}
t_{5}(n) & =t_{5}(-240 m-a)=t_{5}(240 m+a-35) \quad(\text { by Equation }(16)) \\
& \equiv 0(\bmod m)
\end{aligned}
$$

and so $t_{5}(n+240 m) \equiv t_{5}(n)(\bmod m)$. 
(5) $n \leqslant-240 m-35:$ For $n \equiv 0(\bmod 8)$, using Corollary 11, Equation $(16)$ and $m$ odd, we have

$$
\begin{aligned}
t_{5}( & n+240 m) \\
= & t_{5}(-n-240 m-35) \\
= & \left\|\frac{n^{4}}{737280}+\frac{n^{3}}{18432}+\frac{n^{2}}{4608}-\frac{n}{96}-\frac{653}{10800}\right\|+\frac{n^{3}}{768} m+\frac{5 n^{2}}{128} m+\frac{5 n}{48} m \\
& +\frac{15 n^{2}}{32} m^{2}+\frac{5(5 m-1)}{2} m+\frac{75 n}{8} m^{2}+75 n m^{3}+750 m^{3}+4500 m^{4} \\
= & t_{5}(-n-35)+\frac{4 t^{3}}{6} m+\frac{15 t^{2}}{6} m+\frac{5 t}{6} m+30 t^{2} m^{2}+\frac{5(5 m-1)}{2} m+75 t m^{2} \\
& +600 t m^{3}+750 m^{3}+4500 m^{4} \quad(\text { by substituting } n=8 t) \\
\equiv & t_{5}(n)(\bmod m) \quad(\text { by Lemma } 12 \text { and } 5(5 m-1) \equiv 0(\bmod 2)) .
\end{aligned}
$$

As before, Equation (17) is also satisfied for $n \equiv 1,2,3,4,5,6,7(\bmod 8)$.

If $m$ is odd then Equation (17) is satisfied for any integer $n$ from (1), (2), (3), (4) and (5). Similarly, one can show that if $m$ is even then Equation (18) is satisfied for any integer $n$ by using Lemma 12, Corollary 11 and Equation (16). Therefore, if $m$ is odd $240 \mathrm{~m}$ is a period of the sequence and $L$ divides $240 \mathrm{~m}$, else $480 \mathrm{~m}$ is a period of the sequence and $L$ divides $480 \mathrm{~m}$.

Secondly, we prove that if $m$ is odd then $L=240 m$ else $L=480 m$. This can be verified by a computer program for small $m \leqslant 10$. So here, we prove $L=36 \mathrm{~m}$ for only $m>10$. Let $\lambda=L, L-1, L-2, L-3, L-4, L-5, L-6$ or $L-7$ so that $\lambda \equiv 0(\bmod 8)$.

Since $t_{5}(-7)=t_{5}(-6)=\cdots=t_{5}(4)=0$, we have

$$
t_{5}(\lambda) \equiv t_{5}(\lambda+1) \equiv t_{5}(\lambda+2) \equiv t_{5}(\lambda+4) \equiv 0(\bmod m) .
$$

So, we get $m \mid M$ where

$$
\begin{gathered}
M=\left[t_{5}(\lambda+4)-t_{5}(\lambda)\right]+\left[t_{5}(\lambda+1)-t_{5}(\lambda+2)\right]+\left[t_{5}(\lambda+4)-t_{5}(\lambda+2)\right] . \\
t_{5}(\lambda)=\left\|\frac{\lambda^{4}}{737280}+\frac{\lambda^{3}}{18432}+\frac{\lambda^{2}}{4608}-\frac{\lambda}{96}-\frac{653}{10800}\right\| \\
t_{5}(\lambda+1)=\left\|\frac{\lambda^{4}}{737280}+\frac{13 \lambda^{3}}{92160}+\frac{113 \lambda^{2}}{23040}+\frac{29 \lambda}{480}+\frac{1507}{10800}\right\| \\
t_{5}(\lambda+2)=\left\|\frac{\lambda^{4}}{737280}+\frac{11 \lambda^{3}}{92160}+\frac{77 \lambda^{2}}{23040}+\frac{17 \lambda}{480}+\frac{1507}{10800}\right\| \\
t_{5}(\lambda+4)=\left\|\frac{\lambda^{4}}{737280}+\frac{7 \lambda^{3}}{92160}+\frac{23 \lambda^{2}}{23040}+\frac{\lambda}{480}+\frac{157}{10800}\right\|
\end{gathered}
$$


Hence, from Equation (11), we can write

$$
\begin{gathered}
t_{5}(\lambda+4)-t_{5}(\lambda)<\frac{\lambda^{3}}{46080}+\frac{\lambda^{2}}{1280}+\frac{\lambda}{80}+\frac{3}{40}+1 \\
t_{5}(\lambda+1)-t_{5}(\lambda+2)<\frac{\lambda^{3}}{46080}+\frac{\lambda^{2}}{640}+\frac{\lambda}{40}+1 \\
t_{5}(\lambda+4)-t_{5}(\lambda+2)<-\frac{\lambda^{3}}{23040}-\frac{3 \lambda^{2}}{1280}-\frac{\lambda}{30}-\frac{1}{80}+1
\end{gathered}
$$

and it follows that

$$
M=\left[t_{5}(\lambda+4)-t_{5}(\lambda)\right]+\left[t_{5}(\lambda+1)-t_{5}(\lambda+2)\right]+\left[t_{5}(\lambda+4)-t_{5}(\lambda+2)\right]<\frac{\lambda}{240}-\frac{1}{20}+3 .
$$

Similar to Section 3, we can conclude that $L \geqslant 2880$ for $m>10$ by observing an aperiodic pattern for $\left\{t_{5}(n)(\bmod m)\right\}_{n=-\infty}^{\infty}$ with the aid of a computer program. That is, we have $L \geqslant 2880$ so that $\lambda \geqslant 2880$. From (10), we get

$$
\begin{aligned}
t_{5}(\lambda+1)+t_{5}(\lambda) & =\left\|\frac{8 \lambda^{3}}{92160}+\frac{108 \lambda^{2}}{23040}+\frac{34 \lambda}{480}+\frac{2160}{10800}\right\|+\alpha_{1}, \quad-1 \leqslant \alpha_{1} \leqslant 1 \\
& =\left\|\frac{8 \lambda^{3}}{92160}\right\|+\left\|\frac{108 \lambda^{2}}{23040}\right\|+\left\|\frac{34 \lambda}{480}\right\|+\left\|\frac{2160}{10800}\right\|+\alpha_{2}, \quad-4 \leqslant \alpha_{2} \leqslant 4
\end{aligned}
$$

and

$$
\begin{aligned}
& 2\left(t_{5}(\lambda+2)+t_{5}(\lambda+3)\right) \\
= & 2\left(\left\|\frac{4 \lambda^{3}}{92160}+\frac{54 \lambda^{2}}{23040}+\frac{16 \lambda}{480}+\frac{1350}{10800}\right\|+\beta_{1}\right), \quad-1 \leqslant \beta_{1} \leqslant 1 \\
= & \left\|\frac{8 \lambda^{3}}{92160}+\frac{108 \lambda^{2}}{23040}+\frac{32 \lambda}{480}+\frac{2700}{10800}\right\|+\beta_{2}, \quad-3 \leqslant \beta_{2} \leqslant 3 \\
= & \left\|\frac{8 \lambda^{3}}{92160}\right\|+\left\|\frac{108 \lambda^{2}}{23040}\right\|+\left\|\frac{32 \lambda}{480}\right\|+\left\|\frac{2700}{10800}\right\|+\beta_{3}, \quad-6 \leqslant \beta_{3} \leqslant 6 .
\end{aligned}
$$

Hence,

$$
\begin{aligned}
M & =\left\|\frac{34 \lambda}{480}\right\|-\left\|\frac{32 \lambda}{480}\right\|+\gamma_{1}, \quad-10 \leqslant \gamma_{1} \leqslant 10 \\
& =\left\|\frac{\lambda}{240}\right\|+\gamma_{2}, \quad-11 \leqslant \gamma_{2} \leqslant 11
\end{aligned}
$$

Since $\lambda \geqslant 2880$, we have $M>1$. We have $M<\frac{\lambda}{240}-\frac{1}{20}+3=\frac{\lambda+708}{240}$. This means that $m<\frac{\lambda+708}{240}$ (since $m \mid M$ and $M \neq 0$ ), and it follows that

$$
240 m<\lambda+708 \leqslant L+708
$$


If $m$ is odd then $L$ is a divisor of $240 m$, and so $L=240 m$ by Inequality (19) for any $m>10$. Hence, if $m$ is odd then $L=240 m$. If $m$ is even then $L$ is a divisor of $480 m$, so $L=240 m$ or $480 m$. However, we can get

$$
\begin{aligned}
t_{5}(240 m) & =\left\|4500 m^{4}+750 m^{3}+\frac{25 m^{2}}{2}-\frac{5 m}{2}-\frac{653}{10800}\right\| \\
& =4500 m^{4}+750 m^{3}+\frac{5 m}{2}(5 m-1) \\
& \equiv \frac{5 m}{2}(\bmod m) \\
& \neq \equiv(\bmod m),
\end{aligned}
$$

when $m$ is even. Therefore, if $m$ is even then $L$ can not be $240 m$. This means that if $m$ is even then $L=480 \mathrm{~m}$ and the theorem is proved.

\section{Conclusion}

In this paper we introduce a new sequence $\left\{t_{k}(n)\right\}_{n=-\infty}^{\infty}$ for any given positive integer $k \geqslant 3$ that is a generalization of Alcuin's sequence. We find explicitly a linear recurrence equation and the generating function for $\left\{t_{k}(n)\right\}_{n=-\infty}^{\infty}$. The case $k=3$ is Alcuin's sequence. For the special case $k=4$ and $k=5$, we get simpler formulas for $\left\{t_{k}(n)\right\}_{n=-\infty}^{\infty}$ and investigate the period of $\left\{t_{k}(n)\right\}_{n=-\infty}^{\infty}$ modulo a fixed integer. Also, we get a formula for $p_{5}(n)$, the number of partitions of $n$ into exactly 5 parts.

In [1], Andrews discussed the geometric interpretation of the sequence $\left\{t_{3}(n)\right\}_{n=0}^{\infty}$ corresponding to Alcuin's sequence. For $k=4$, let $a, b, c$ and $d$ be integers satisfying the condition

$$
a+b+c+d=3 n, a+b+c>2 n, a+b+d>2 n, b+c+d>2 n \text { and } a+c+d>2 n .
$$

If this is case $a, b, c, d<n$, and since

$$
t_{4}(3 n)=p_{4}(n)
$$

via the bijection

$$
(a, b, c, d) \longleftrightarrow(n-a, n-b, n-c, n-d),
$$

we conclude that $t_{4}(3 n)$ counts the number of different $a, b, c, d$ integers which satisfy Condition (20). We wonder what kind of geometric interpretation does $\left\{t_{4}(n)\right\}_{n=-\infty}^{\infty}$, or more generally $\left\{t_{k}(n)\right\}_{n=-\infty}^{\infty}$ for $k \geqslant 4$, represent? 


\section{References}

[1] E. G. Andrews. A note on partitions and triangles with integer sides. Amer. Math. Monthly, 86:477-478, 1979.

[2] N. Biggs. Discrete Mathematics, Oxford University Press, 2002.

[3] D. J. Bindner and M. Erickson. Alcuin's Sequence. Amer. Math. Monthly, 119:115$121,2012$.

[4] D. Olivastro. Ancient Puzzles, Bantam Books, New York, NY, 1993.

[5] http://mathworld.wolfram.com/PartitionFunctionP.html (as seen on October 11, 2012). 